\title{
O processo de construção da enfermagem obstétrica: uma revisão narrativa
}

\author{
The process of constructing midwifery nursing: a narrative review \\ El proceso de construcción de la enfermería obstétrica: una revisión narrativa
}

Raiza Amanda Gonçalves de Souza1*, Tayná de Sousa Alencar da Silva1, Thamires Bezerra Almeida Brito ${ }^{1}$, Cristiane da Silva Nascimento ${ }^{1}$, Luana Maria Angelo dos Santos ${ }^{2}$, Maria Thamylle Ramos Nery ${ }^{1}$, José Eduardo Pereira Alcântara ${ }^{1}$, Eulária Araújo de Souza ${ }^{1}$, Bruna Emanuele Barreto Pinheiro², Joab Gomes da Silva Sousa'.

\section{RESUMO}

Objetivo: Elucidar o processo de construção do conhecimento formalizado da enfermagem obstétrica. Revisão bibliográfica: Os saberes empíricos relativos à gestação e ao processo de parto, eram atribuídos aos cuidados de parteiras, elas eram responsáveis pela atenção pré-parto, cuidados imediatos ao recémnascido e ao corpo feminino no pós-parto, nesse cenário o processo de parturição era considerado fisiológico e natural. Esse espaço foi sendo transformado sob influência do modelo biomédico, mudando o cenário de parto para um onde há internações, intervenções e medicalização do parto e tais procedimentos estão associados a desfechos trágicos. Assim, observa-se a crescente especialização de enfermeiros em obstetrícia, atuando na criação de tecnologias e ferramentas de cuidado, objetivando a promoção da saúde e a disseminação das boas práticas de atenção ao parto. Considerações finais: Observa-se a importância histórica do profissional que ao longo do tempo, foi se especializando e formalizando os seus saberes e hoje são protagonistas na promoção das boas práticas de atenção ao parto e nascimento e a saúde da mulher.

Palavras-chave: Enfermagem obstétrica, História da enfermagem, Parto humanizado.

\begin{abstract}
Objective: To elucidate the process of construction of formalized knowledge of the obstetric nursing. Bibliographic review: The empirical knowledge related to pregnancy and childbirth process was attributed to the care of midwives, who were responsible for the prepartum care, immediate care to the newborn and to the female body in the postpartum period, in this scenario the parturition was considered physiological and natural. This space has been transformed under the influence of the biomedical model, changing the childbirth scenario to one where there are hospitalizations, interventions, and medicalization of childbirth, and such procedures are associated with tragic outcomes. Because of that, there is a growing specialization of nurses in obstetrics, working on the creation of technologies and care tools, aiming at health promotion and the dissemination of good practices in the childbirth care. Final considerations: The historical importance of professionals who, over time, have specialized and formalized their knowledge is observed, and today they are protagonists in the promotion of good practices for the childbirth care and for women's health.
\end{abstract}

Keywords: Obstetric nursing, History of nursing, Humanizing delivery.

\section{RESUMEN}

Objetivo: Dilucidar el proceso de construcción del conocimiento formalizado en enfermería obstétrica. Revisión bibliográfica: El conocimiento empírico relacionado con embarazo y el proceso del parto se atribuía a atención de las matronas, encargadas de los cuidados previos al parto, del cuidado inmediato del recién nacido y del cuerpo femenino en el posparto; Hasta aquí, el proceso del parto se consideraba fisiológico y natural. Este espacio se ha transformado, por influencia del modelo biomédico, haciendo con que el parto sea seguido de una hospitalización, intervenciones y medicalización, siendo procedimientos asociados a

1 Universidade Regional do Cariri (URCA), Crato - CE. *E-mail: raiza.amanda@urca.br

2 Centro Universitário de Juazeiro do Norte (UNIJN), Juazeiro do Norte - CE. 
resultados trágicos. Así, se observa la creciente especialización de los enfermeros en obstetricia, actuando en la creación de tecnologías y herramientas de atención, con objetivo de promover la salud y diseminar buenas prácticas de atención al parto. Consideraciones finales: Se observa la importancia histórica del profesional que se fue especializando y formalizando sus conocimientos y hoy es protagonista en promover buenas prácticas de atención al parto y nacimiento y a salud de la mujer.

Palabras clave: Enfermería obstétrica, Historia de la enfermería, Parto humanizado.

\section{INTRODUÇÃO}

O processo de inserção da Enfermagem Obstétrica (EO) remete ao princípio do nascimento, momento unicamente feminino, o planejamento e o parto eram tradições das mulheres que ganhavam seus filhos em casa, assistidas por mulheres conhecidas por parteiras, estas sem diploma ou ensino formal, habilitadas com à experiência passada de mãe para filha, ou com os próprios partos. A sua atuação era caracterizada por sua prática baseada em experiências individuais, guiadas pela intuição e guardadas pela fé (CARREGAL FAS, et al., 2020).

Além da assistência direta ao parto, essas mulheres eram responsáveis pelos cuidados imediatos ao recém-nascido e ao corpo feminino no pós-parto, o processo de parturição era considerado fisiológico e natural, um momento puramente feminino sendo atribuído às mulheres o protagonismo (OLIVEIRA VJ e PENNA CMM, 2018).

Porém, entre os séculos XIV e XVIII, foi realizada uma campanha contra à assistência prestadas por mulheres leigas, sendo este um marco histórico que iniciou o declínio da atuação das parteiras. Sendo imputado a médicos assistência ao parto e nascimento, levando à inserção de medicalização e instrumentalização do parto, constituindo à institucionalização do parto associando-o as rotinas cirúrgicas. Todavia, as parteiras não se extinguiram por conta de tal campanha, mas, regulamentaram o seu trabalho, pois, em 1902 na Inglaterra, foi criado um conselho de classe, com o objetivo de respaldar o exercício profissional desta categoria (PALHARINI LA e FIGUEIRÔA LSDM, 2018).

Observa-se que à inserção do médico no cenário de parto junto com os avanços tecnológicos e ações intervencionistas, os quais contribuíram para a construção da ideia de parto como processo patológico, que requer procedimentos cirúrgicos, que consequentemente elevou índice de mortalidade maternal, hoje no Brasil é refletido no que chama-se de epidemia de cesarianas. Motivo pelo qual fez necessário a criação de políticas públicas que revisem e apliquem as melhores formas da assistência a ser prestada para a redução de danos, a epidemia de cesarianas no Brasil é reflexo de uma cultura intervencionista que trabalha sob a medicalização e o tratamento do parto, o que está intimamente relacionado as elevadas taxas de morbimortalidade materna e perinatal no país. (BATISTA M e RISSIN A, 2018; MACHADO AC, et al., 2020).

E por tal, consequentemente houve a necessidade de reformulação do modelo de formação dos profissionais de saúde, objetivando o seu reflexo no sistema de saúde, sendo a enfermagem obstétrica uma das alternativas para esta reformulação, visto a capacitação destes profissionais na assistência ao parto normal de risco habitual, centrada na preservação da autonomia da mulher no processo de parto, valorizando o nascimento seguro e livre de intervenções cirúrgicas e medicamentosas desnecessárias (SANTOS GG e PAÇO JAO, 2020).

Visto as mudanças socioculturais advindas pelo processo já descrito e as consequências associadas a este, a busca por mudanças na assistência, nas políticas, nos serviços e nas concepções agora criadas, tornou-se um objetivo de âmbito global, como citado no Objetivo do Desenvolvimento Sustentável (ODS)-3, elaborado pela Organização Mundial da Saúde (OMS) e tomados pelos países associados, o qual define como meta a redução da mortalidade maternal global e como estratégia, estimula a formalização e o exercício professional de parteiras, obstetrizes e enfermeiras obstetras (RAMOS WMA, et al., 2018).

Deste modo, objetivou-se elucidar o processo de construção do conhecimento formalizado da enfermagem obstétrica, tendo em vista a importância da sua atuação para a promoção da saúde da mulher, sua dignificação, humanização do cenário de parto e redução de eventos trágicos perinatais. 


\section{REVISÃO BIBLIOGRÁFICA}

Torna-se possível observar, dentro da linha do tempo, que o processo de Trabalho de Parto (TP) partiu de um processo íntimo feminino, livre de intervenções médicas, onde mulheres desempenhavam a função de assistência ao parto, as parteiras, que auxiliavam no momento de nascimento e pós-parto, baseadas nos conhecimentos empíricos adquiridos entre gerações, sendo substituído pelo hoje conhecido como o modelo mecaniscista-biomédico, reduzindo a atuação da parteira aos limites definidos pelos médicos em contrapartida estabelecendo a necessidade de hospitalização, medicalização e rotinas cirúrgicas para o processo parturitivo (ZIRR GM, et al., 2019).

A implementação da enfermagem obstétrica está em consonância com as considerações da OMS considerando o seu caráter formativo menos intervencionista, com práticas humanizadas e holísticas sendo estes apropriados para o acompanhamento das gestações de risco habitual, sendo essenciais para a promoção da saúde materna, como preconiza os Objetivos do Desenvolvimento Sustentável (ODS) em especial o ODS 3, que versa sobre a redução da mortalidade materna (OLIVEIRA TR, et al., 2021).

Assim a enfermagem obstétrica atua na sustentação do direito da mulher na decisão de como se seguirá o seu TP, assim como no fortalecimento da política de humanização, promoção do empoderamento feminino, e centralização da mulher com protagonista na gestação e no ato de parir (RAMOS WMA, et al., 2018).

Nessa conjuntura, em 1984 ouve a criação do Programa de Assistência Integral à Saúde da Mulher (PAIMS), o qual inclui ações preventivas, tratamento e ações de recuperação durante à assistência pré-natal, parto e puerpério, além das ações voltadas para o climatério, o que representa uma quebra no conceito da saúde materno-infantil como única forma de cuidar da mulher (OLIVEIRA RS, et al., 2019).

Nesse sentido, surgiram os primeiros programas voltados a saúde materno-infantil, elaborados nas primeiras décadas do século XX, porém ainda não compreendiam todas facetas do cuidado a gestante e a gestação em todos os seus âmbitos, apresentavam uma visão reducionista voltada apenas a fisiologia da mulher e do parto, não sendo capaz de dissociar a mulher do seu papel social de mãe, o que tornava limitado o conceito de cuidado integral a saúde da mulher além do processo parturitivo (MACHADO AC, et al., 2020).

Esse processo em suma, configura-se uma transformação social, cultural e política no que se refere à assistência à mulher, e hoje $\mathrm{a} E O$, assumem um importante papel no acompanhamento e realização de partos mediante formação prática e teórica através do conhecimento científico. Essa categoria busca a melhoria do sistema de saúde e promoção da saúde do binômio mãe e filho através dos seus cuidados, não desprezando as singularidades do Ser Mulher (OLIVEIRA RS, et al., 2019).

A atuação da parteira com o passar do tempo sofreu bastante interferências, mas hoje, graças a organização em classes, de conselhos e sindicados, houve a elaboração de um currículo de formação para esta profissão, assim podemos identificar sua essência no exercício profissional de enfermeiros Obstetras e Obstetrizes, os quais atuam também no parto domiciliar planejado, com uma trajetória profissional focada no aprimoramento contínuo e aprimoramento da prática, conferindo experiência, qualidade e segurança ao exercício profissional com embasamento cientifico, além da estímulo às práticas de parteiras tradicionais em comunidade que ainda mantiveram essa tradição (OLIVEIRA TR, et al., 2021; OLIVEIRA RS, et al., 2019).

Por meio dessa breve análise histórica tornou-se possível observar a importância dos cursos de aprimoramento e capacitação profissional da especialização da enfermagem em obstetrícia e a sua contribuição na área da assistência humanizada, respeitando o caráter fisiológico do parto e o empoderamento feminino, assim fica evidente a necessidade e planejamento de novas estratégias que promovam a valorização e inserção dos enfermeiros obstetras, visando o reconhecimento profissional no mercado de trabalho atual (SANTOS FAPS, et al., 2019).

As formas de aperfeiçoamento em enfermagem obstétrica, são compostos por atividades teórico-práticas além de treinamento em serviços especializados como as maternidades e hospitais referenciais. $O$ objetivo envolve a promoção da atenção integral à mulher e o ciclo gravídico-puerperal, abraçando todos os âmbitos do cuidado a saúde e promovendo o protagonismo da mulher, observando as singularidades femininas, de forma técnica, humanizada e ética, fundamentados na prática baseada em evidências (SILVA GF, et al., 2020). 
Assim o especialista em obstetrícia deve oferecer suporte à mulher e sua família durante a gravidez, de maneira humanizada, ética, responsável, equânime, valorizando e respeitando o protagonismo da mulher, pois este profissional é tecnicamente habilitado para o desenvolvimento das atividades supracitadas além da tomada de decisão imediata, desde o pré-natal qualificado, um parto seguro e uma assistência presente no pós-parto (NASCIMENTO ER, 2020).

Porém a inserção do EO na realidade brasileira ainda é um desafio a ser superado nas instituições de cuidado em obstetrícia, e um dos pontos apontados como frágeis nesse processo é a estranheza da equipe em compreender a importância da atuação do EO, e a falta de delimitação clara do papel de cada profissional e a dificuldade na interação multiprofissional (NASCIMENTO ER, 2020; PROGIANTI JM, et al., 2018).

Outro desafio para a atuação dos EO no exercício de suas práticas é a ausência de infraestrutura, espaço e equipamentos para a atuação e promoção das práticas e atividades holísticas, a falta de um espaço próprio que favoreça a melhoria do serviço, o que se tornou bastante evidente nos dias atuais com o trabalhar dos EO em meio a pandemia de COVID-19 (COSTA TP, et al., 2021).

Destaca-se que a atuação da residência em enfermagem obstétrica eleva a qualidade da assistência, visto a prática exercida por profissionais com uma bagagem acadêmica mais robusta fundamentada na prática, preparados para situações do cotidiano, ou seja, promove mudanças nos cenários de atividade onde estão inseridos e sua implementação contribui com a promoção do cuidado integral, mesmo frente os desafios enfrentados (MACIEL VS e DORNFELD D, 2019).

A estrutura de ensino-formação dos EO embasada na política da humanização, sob olhar holístico e estímulo ao desenvolvimento de ações não farmacológicas, redução do uso indiscriminado de medicamentos e cesarianas, reflete um relativo impacto no campo de prática, onde o modelo consolidado é o biomédico e toda a equipe envolvida já está estruturada sob esse modelo, representando um desafio para mudanças (PEREIRA SB, et al., 2018).

Desse modo fica evidente o caráter cientifico que a enfermagem obstétrica vem conquistando, e juntamente a este, observa-se o desenvolvimento de momentos, ferramentas e tecnologias em saúde, visando a promoção da saúde da mulher em meio ao processo de gestação. O Enfermeiro Obstetra tem liberdade técnica e legal para o desempenho profissional voltado ao pré-natal, parto e puerpério, sendo este capaz de potencializar a qualidade da assistência humanizada e holística em todos estes cenários (SOUZA MCT, et al., 2019).

Nesse sentido a melhoria do ensino em enfermagem com a especialização na obstetrícia deve estar em sintonia com as melhoras no serviço de assistência, refletindo em mudanças nos setores de saúde onde recebem EO, em virtude da inclusão deste profissional no cenário em questão, contribuindo com a conquista dos objetivos institucionais e globais (CARDOSO RF, et al., 2019).

Observando a atuação do EO durante as consultas de pré-natal, percebe-se que são executadas algumas demandas padrões como vacinação da gestante, solicitação de exames laboratoriais de rotina, exames físicos, dentre outros que buscando a prevenção de doenças que acarretem danos a mãe e/ou filho, assim como identificação precoce de alterações e intervenção imediata quando necessário (OLIVEIRA RS, et al., 2019).

Durante o pré-natal, as atividades de educação em saúde, organizadas por enfermeiros, tornam-se uma prática frequentes, pois as atividades educativas, em suma são estruturadas como espaço de discussão sobre questões relevantes para a promoção da assistência qualificada para a mulher, família e filhos, esse ambiente de discussão favorece a troca de conhecimentos entre a equipe de saúde e as gestantes, no pré-natal, as iniciativas educativas visão preparar a gestante para a vivência do ciclo gravídico-puerperal (SIMÃO AMS, et al., 2019).

As atividades educativas em saúde são realizados por enfermeiros que buscam o esclarecimento de dúvidas das gestantes assim como a apresentações de temas pertinentes a saúde, como as mudanças fisiológicas advindas da gestação, hipertensão e outras patologias, amamentação, cuidados com o recémnascido, assim como as possibilidades de vias de parto, o direito ao acompanhante, apresentação das terapias complementares que podem ser utilizadas na redução do estresse, inclusão dos familiares dentre 
outros temas, essas discussões durante o pré natal tem consequências benéficas na promoção da autonomia e segurança maternal (CARDOSO RF, et al., 2019).

O processo de educação em saúde é imprescindível para a compreensão da dinâmica familiar em que vive a mulher, levantando os determinantes sociais de risco para ela e sua gestação e através destes elaborar as melhores medidas de intervenções, como demonstrou um estudo de Oliveira TR, et al., (2021), onde abordou o letramento sobre dependência de álcool e outras drogas com gestantes, e concluíram a fragilidade económica e social das pessoas, concluindo que quanto mais socialmente vulnerável está a mulher, maior os riscos relacionados à falta de conhecimento sobre pré-natal, parto e pós-parto, sendo este, um importante desafio na promoção da educação em saúde por parte do enfermeiro na atenção primária (OLIVEIRA RS, et al., 2019).

A educação em saúde torna a mulher consciente sobre as mudanças fisiológicas, emocionais, psicológicas e sociais que passará, tornando-a autônoma nas escolhas sobre as questões relativas à sua gestação, assim como a livre escolha no seu plano de parto, ciência dos seus direitos, e as possibilidades de emprego das Práticas Integrativas e Complementares em Saúde (PICs) durante o TP propriamente dito. O enfermeiro obstetra é habilitado para estas ações educativa, assim como identificar falhas nesse processo e resolvê-las em tempo hábil, sanar dúvidas, orientar quando a práticas diárias, vícios, hábitos nocivos, alimentação e direitos, é tornar a mulher participante ativa no cuidado, promovendo sua autonomia, segurança e conforto (CARDOSO RF, et al., 2019).

Durante as consultas de pré-natal, os profissionais que acompanham a mulher, devem elaborar, junto com a mesma, o plano de parto com as recomendações das gestantes, direcionando-os para os serviços de atenção ao parto, proporcionando a interdisciplinaridade no cuidado, com vinculação da equipe multiprofissional, implementando a visão de promoção dos desejos planejados pela mulher durante o seu prénatal, garantindo saúde e humanização do parto e nascimento (SOUZA MCT, et al., 2019).

Durante O TP o enfermeiro obstetra desempenha atividades ímpares na unidade hospitalar e/ou casas/centros de partos, onde os cuidados assistências estão em sintonia com as atividades gerenciais da unidade, foi observado que o planejamento da prática assistencial, a liderança da equipe de enfermagem juntamente com a ânsia por conhecimentos, e capacitações sobre melhoria das práticas no cuidado obstétrico, e estas ações podem propiciar significativos resultados na assistência prestada além da potencialização da autonomia do enfermeiro na unidade (BERNARDY CCF, et al., 2019).

Na prática assistencial a enfermagem atua na promoção da segurança e conforto, utilizando de uma escuta ativa e atenciosa as demandas expostas pelas gestantes, onde a criação de vínculo, torna-se necessário, para a identificação de necessidades, ansiedades, alterações nas gestantes e parturientes, e este cenário favorece o protagonismo do EO em atuar na promoção da humanização direcionada as demandas apontadas, uma assistência sem preconceitos, baseada no respeito, solidariedade, incentivo, com utilização mínima de intervenções invasiva (NASCIMENTO ER, 2020).

A atuação do EO na assistência ao TP com a participação ativa da mulher deve ser baseada no dinamismo entre as partes, onde haja troca de saberes com a gestante, incorporando-os a fundamentos científicos ao mesmo tempo que preserva a autonomia da mulher. $\mathrm{O}$ acolhimento, e na consulta de enfermagem adequados possibilita O EO identificar fatores estressantes, dor, medo, ansiedades e inseguranças e a parir destes diagnósticos prévios, torna possível o desenvolvimento dos cuidados de enfermagem (DULFE PAM, et al., 2021)

Graças aos cuidados ligados a política de humanização, a assistência de enfermagem está relacionada a redução de medidas intervencionistas como o uso indiscriminado de medicamento, realização de episiotomia, tricotomia pubiana, amniotomia, posição litotómica, uso de cateter periférico, dentre outros, e graças a redução destas práticas há consequentemente a redução de riscos associados ao trabalho de parto (NASCIMENTO ER, 2020).

Durante o TP e parto, estressores fisiológicos são esperados como a dor, ansiedade, contrações e medo, e a enfermagem deve encontrar modos de reduzir esses estressores, e a promoção do vínculo materno pelo filho, como por exemplo o toque pele a pele, a amamentação imediata, implementação da hora de ouro, 
garantia do direito a acompanhante, no pós-parto, deve-se prestar cuidados ao recém-nascidos com respeito a amamentação, vacinação, registros, avaliando a presença de icterícia e realização de exames (LIMA MFG, et al., 2018).

O cuidado a mulher no pós-parto imediato e puerpério não deve ser algo negligenciado, pois o monitoramento do bem-estar e identificação de possíveis complicações devem ser constantes na atividade do EO e demais da equipe, pois, complicações nesse período podem colocar a vida da mulher em risco, e o EO deve estar habilitado a atuar frente as intercorrências no pós-parto, garantindo agilidade e segurança na identificação e intervenção da complicação (MACIEL VS e DORNFELD D, 2019).

Musicoterapia, aromoterapia, acupuntura, massagem, banhos mornos, dentre outras, fazem parte das conhecidas Práticas Integrativas e Complementares (PIC's) que podem ser aplicadas durante o TP e puerpério, pois promovem uma assistência holística para estas mulheres, estas apresentam importantes resultados no relaxamento, alívio da dor e ansiedade, redução do tempo de TP, melhoria do conforto, redução de trauma perineal, regulação das contrações uterinas, promoção da calma e coragem para as mulheres. Porém, mesmo com inúmeros benefícios estas práticas não são amplamente difundidas entre as maternidades brasileiras, o que torna importante a capacitação de enfermeiros acerca das PIC's (SILVA ADV, et al., 2020; BERNARDY CCF, et al., 2019).

A presença do EO em todas as fases do ciclo gravídico-puerperal, como simploriamente foi apresentado, configura a inserção estratégica de um profissional capacitado para a assistências à mulheres dentro deste perfil, o fato de ter um EO desde o pré-natal faz com que haja um olhar clínico sobre o cuidado da mulher, identificando fragilidades e vulnerabilidades desde o início da gestação, e ao direcionar o cuidado para a redução destas, buscar-se-á que elas sejam sanadas, e assim os riscos que outrora agiriam como cascata que culminariam na ocorrência de evento materno trágico ou traumático para a mulher, seu filho e seus familiares não mais existiriam (DULFE PAM, et al., 2021)

Evidentemente a autonomia do Enfermeiro obstetra, tem respaldo legal, jurídico e ético para ser efetivada nos serviços, além de recomendações internacionais acerca dos seus resultados positivos, todavia, estudos mostram que há dificuldades claras na inserção do EO, e citam a insuficiência de enfermeiros especialistas na salas de parto, a ausência de espaço para o desenvolvimento de autonomia do profissional, além da existência de influência político-partidária na gestão em saúde, e falhas internas no processo de assistência entre os membro da equipe multiprofissional, que estabelecem barreiras para a plena implementação das atividades do enfermeiro especializado na área (MAGALHÃES TTS e TAFFENER VBM, 2020).

Enfermagem Obstétrica tem um encargo histórico de cuidado que Ihe atribui uma caminhada longa de conquista de espaço, atrelada diretamente aos cuidados perinatais, hoje em dia é uma importante ferramenta para a disseminação das boas práticas de atenção ao parto e nascimento. A especialização em enfermagem obstétrica é uma das formas de capacitação profissional que tem ganhado destaque, visto o seu modelo de formação inserido nos serviços de saúde, porém ainda enfrenta importantes impasses para a sua mais ampla implementação nos mais diversos cenários e atenções (SANFELICE CFO, 2020).

\section{CONSIDERAÇÕES FINAIS}

Assim, nos dias atuais, o empoderamento e a visibilidade da categoria profissional da enfermagem obstétrica fortalecem o aprimoramento e aquisição de habilidades e segurança técnica, além de estimular a criação de estratégias e ferramentas que contribuam para a superação dos impasses gerados pelos modelos adotados, o que historicamente foi atribuído as atividades das parteiras hoje em dia toma valor nas evidências cientificas e são validadas na pratica assistencial assim como nas recomendações internacionais. Em meio a todos esses entrelaces historicamente construídos, observa-se o papel do enfermeiro atuando na promoção de saúde e aplicação das boas práticas de atenção à saúde e aplicação destes princípios na atenção maternal e infantil, retomando os conceitos históricos atribuídos as parteiras porém, nos dias atuais, esta categoria passa por especializações com fundamentação teórica e prática, os tornando habilitados para atuar na elaboração de ferramentas, protocolos, princípios de cuidado e boas práticas de atenção, intervindo na promoção da saúde da mulher, dignificação do Eu e redução dos eventos trágicos perinatais. 


\section{REFERÊNCIAS}

1. BATISTA M, RISSIN A. WHO and the epidemic of cesarians. Revista Brasileira de Saúde Materno Infantil, 2018; 18(1): 3-4.

2. BERNARDY CCF, et al. Práticas Integrativas e Complementares em Saúde em uma maternidade paranaense. Revista Guará, 2019; 12(1): 55-65.

3. CARDOSO RF. Educação em saúde na assistência pré-natal: revisão de literatura. Revista Eletrônica Acervo Saúde, 2019; 23(1): 1-8.

4. CARREGAL FAZ, et al. Resgate histórico dos avanços da Enfermagem Obstétrica Brasileira. Hist enferm Rev eletrônica, 2020; 11(2): 123-32.

5. COSTA TP, et al. Os desafios da enfermagem obstétrica no início da pandemia da COVID-19 no Estado do Pará. Research, Society and Development, 2021; 10(3): 1-10.

6. DULFE PAM, et al. Nurse-midwives reconfiguring care in the scope of labor and births in COVID-19 times. Revista Brasileira de Enfermagem, 2021; 74( Suppl 1): 1-8.

7. LIMA MFG, et al. Developing skills learning in obstetric nursing: approaches between theory and practice. Revista Brasileira de Enfermagem, 2017; 70(5): 1054-1060.

8. MACHADO AC, et al. Transformações da identidade feminina ao tornar-se mãe. PsicolArgum. 2020; 38(99): 66-87.

9. MACIEL VS, DORNFELD D. A inserção da enfermeira obstétrica na Assistência hospitalar ao parto. Enferm. Foco, 2019; 10(4): 148-152.

10. MAGALHÃES TTS, TAFFENER VBM. Dificuldades para a atuação autônoma do enfermeiro obstetra no Brasil. REVISA, 2020; 9(4): 685-97.

11. NASCIMENTO ER. Desafios da assistência de enfermagem ao parto humanizado. Caderno De Graduação - Ciências Biológicas e da Saúde, 2020; 6(1): 141-6.

12. OLIVEIRA RS, et al. As parteiras tradicionais e a medicalização do parto na região rural do Amazonas. Sexualidad, Salud y Sociedad, 2019; 33(1): 79-100.

13. OLIVEIRA TR, et al. Assistência ao parto domiciliar planejado: trajetória profissional e especificidades do cuidado da enfermeira obstétrica. Texto Contexto Enferm, 2021; 29(1): 1-14.

14. OLIVEIRA VJ, PENNA CMM. Every birth is a story: process of choosing the route of delivery. Rev Bras Enferm., 2018; 71(Suppl 3): 1228-36.

15. PALHARINI LA, FIGUEIRÔA LSFM. Gênero, história e medicalização do parto: aexposição "Mulheres e práticas de saúde". História, Ciências, Saúde - Manguinhos, 2018; 25(4): 1039- 1061.

16. PEREIRA SB, et al. Good practices of labor and birth care from the perspective of health professionals. Revista Brasileira de Enfermagem, 2018, 71(suppl 3): 1313-1319.

17. PROGIANTI JM, et al. Precarização do trabalho da enfermeira obstétrica. Rev enferm UERJ, 2018; 26(1): 1-7.

18. RAMOS WMA, et al. Contribuição da enfermeira obstétrica nas boas práticas da assistência ao parto e Nascimento. Rev Fund Care, 2018; 10(1): 173-179.

19. SANFELICE CFO. Curso de aprimoramento para enfermeiras obstétricas do Projeto Apice On: relato de experiência. Esc Anna Nery, 2020;24(2): 1-7.

20. SANTOS FAPS, et al. Autonomy for obstetric nurse on low-risk childbirth care. Revista Brasileira de Saúde Materno Infantil, 2019; 19(2): 471-479.

21. SANTOS GG, PAÇO JAO. Visão e atuação humanizada de estudante do curso de Especialização em Enfermagem Obstétrica no parto normal. Glob Acad Nurs, 2020; 1(1): 1-7.

22. SILVA ADV. The benefits of integrative and complementary practices in childbirth work. Research, Society and Development, 2020; 9(7): 1-16.

23. SILVA GF, et al. A formação na modalidade residência em enfermagem obstétrica: uma análise hermenêuticodialética. Escola Anna Nery, 2020; 24(4): 1-8.

24. SIMÃO AMS, et al. Management of prenatal nursing care at a Health Center in Angola. Rev Bras Enferm., 2019; 72(Suppl 1): 129-36.

25. SOUZA MCT, et al. A Importância do Vínculo Profissional Atribuída por Gestante. Id on Line Rev. Mult. Psic., 2019; 13(46): 938-945.

26. ZIRR GM, et al. Autonomia da mulher no trabalho de parto: contribuições de um grupo de gestantes. REME - Rev. Min. Enferm., 2019; 23(1): 1-7. 